\title{
Absence of Discrete Spectrum in Highly Negative Ions ${ }^{\star}$
}

\author{
Mary Beth Ruskai ${ }^{\dagger}$ \\ The Rockefeller University, New York, NY 10021 USA
}

\begin{abstract}
Let $H_{N}$ be the Hamiltonian for the Coulomb system consisting of $N$ particles of like charge in the field of a fixed point charge $Z$. We show that if the particles are bosons, then $H_{N}$ has no discrete spectrum when $N \geqq N_{0}=c Z^{2}$ for some constant $c$. If the particles are fermions, then $H_{N}$ is bounded below uniformly in $N$. These results can be extended to molecules and to other power law potentials.
\end{abstract}

\section{Introduction}

Let $H_{N}$ be the Hamiltonian

$$
H_{N}(W, Z)=-\sum_{j=1}^{N} \Delta_{j}-\sum_{j=1}^{N} Z r_{j}^{-1}+\sum_{j<k} W r_{j k}^{-1} .
$$

When $W=1, H_{N}$ is the Hamiltonian of $N$ charged particles in the field of an infinitely heavy nucleus of charge $Z$. If these particles are fermions and $Z \geqq N+1$, so that $H_{N}(1, Z)$ is the Hamiltonian for a negative ion, it is known $[1-3,18]$ that $H_{N}$ has only finitely many bound states. However, very little is known about the precise number of bound states. When $N=2$, Hill $[4,5]$ has shown that $H_{2}(1,1)$ which is the Hamiltonian for $\mathrm{H}^{-}$, has precisely one bound state in the sector of natural parity; Grosse and Pittner [6] have shown that $H^{-}$has precisely three degenerate bound states in the sector of unnatural parity. Hill's results can be extended to show that $\mathrm{H}^{--}$has no bound states [7], but Hill's techniques are unlikely to be suitable for $N$ much larger than 3 or 4 . All other methods known for estimating the number of bound states of multi-particle systems are either very specialized or very weak $[8-10]$.

In this paper we show that for a system of $N$ charged bosons, $H_{N}(W, Z)$ has no discrete spectrum when $N$ is sufficiently large. Then the only possible bound states are eigenvalues imbedded in the continuum. Because our method of proof uses smoothing functions which need not leave a given symmetry subspace invariant,

* Research supported by the National Science Foundation, MCS78 -20455 USA

$\dagger$ On leave from Department of Mathematics, University of Lowell, Lowell, MA O1854 USA 
we do not prove absence of bound states in each symmetry subspace, nor do we prove absence of discrete spectrum in the antisymmetric subspace corresponding to fermions. However, we believe that fermions also have no discrete spectrum for large $N$ and discuss the extent to which our proof is valid for fermions in Sect. III. Furthermore, we can show that the Hamiltonian $H_{N}$ is bounded below uniformly in $N$ for fermions. Thus, at worst, the fermion binding energy can be made arbitrarily weak by making $N$ sufficiently large.

For convenience we scale $H_{N}(W, Z)$ and consider instead

$$
H_{N}(\omega)=-\sum_{j=1}^{N} \Delta_{j}-\sum_{j=1}^{N} r_{j}^{-1}+\sum_{j<k} \omega r_{j k}^{-1},
$$

where $\omega=W Z^{-1}$. Let $\mathscr{D}\left(H_{N}\right)$ be a core for $H_{N}(\omega)$ with the following property: Whenever $F$ is a bounded $C^{2}$ function and $\Psi$ is in $\mathscr{D}\left(H_{N}\right)$, then $F \Psi$ is also in $\mathscr{D}\left(H_{N}\right)$. Note that $\mathscr{D}\left(H_{N}\right)$ is invariant under multiplication by smoothing functions, but the functions $F$ need not have any properties which guarantee that a particular symmetry subspace of $\mathscr{D}\left(H_{N}\right)$ is also invariant. In particular, $F \Psi$ need not have the same permutational symmetry as $\Psi$. We now define

$$
\varepsilon_{N}=\inf _{\Psi \text { in } \mathscr{D}} \frac{\int \Psi H_{N}(\omega) \Psi d x}{\|\Psi\|^{2}} .
$$

one can similarly define $\varepsilon_{N}^{+}$and $\varepsilon_{N}^{-}$if the infimum is taken over $\Psi$ in the symmetric and antisymmetric subspaces $\mathscr{D}^{+}$and $\mathscr{D}^{-}$respectively. We can now state our main result as:

Theorem 1. For every fixed $\omega$, there is an $N_{0}$ such that $N \geqq N_{0}$ implies $\varepsilon_{N}=\varepsilon_{N-1}$. We will prove Theorem 1 by showing that for sufficiently large $N$

$$
\int \Psi_{N} H_{N}(\omega) \Psi_{N} d x>\varepsilon_{N-1}\|\Psi\|^{2}
$$

for all $\Psi$ in $\mathscr{D}\left(H_{N}\right)$. This implies that $\varepsilon_{N} \geqq \varepsilon_{N-1}$. Since $\varepsilon_{N} \leqq \varepsilon_{N-1}$ always, we conclude that $\varepsilon_{N}=\varepsilon_{N-1}$. Furthermore, it follows from the Hunziker-van Winter-Zhislin (HVZ) theorem [11-14] that the essential spectrum of $H_{N}(\omega)$ begins at $\varepsilon_{N-1}$. Therefore $H_{N}(\omega)$ has discrete spectrum if and only if $\varepsilon_{N}<\varepsilon_{N-1}$, so that our theorem implies that $H_{N}(\omega)$ has no discrete spectrum for $N \geqq N_{0}$. Since (3) is a strict inequality, we can also conclude that $\varepsilon_{N}$ is not an eigenvalue of $H_{N}(\omega)$ for sufficiently large $N$. Our method of proof will also show that $N_{0} \leqq c Z^{2}$ for some constant $c$.

We emphasize again that our proof requires smoothing functions $F$ which do not leave the symmetric and antisymmetric domains $\mathscr{D}^{ \pm}$invariant. Thus the above statements are valid only if we impose no permutational symmetry restrictions on $\Psi$. However, we can extend Theorem 1 and the discussion in the preceding paragraph to bosons as follows: Suppose $\varepsilon_{N}=\varepsilon_{N-1}$ and $\varepsilon_{N-1}$ is not an eigenvalue of $H_{N-1}(\omega)$. Then the HVZ theorem implies that $\varepsilon_{N-1}=\varepsilon_{N-2}$. Therefore, there is an $N^{\prime}$ so that $\varepsilon_{N}=\varepsilon_{N-1}=\ldots=\varepsilon_{N^{\prime}}$ and $\varepsilon_{N^{\prime}}$ is an eigenvalue of $H_{N^{\prime}}(\omega)$. It is well known [14] that the ground state of $H_{N^{\prime}}(\omega)$ is unique and positive, which implies that the ground state is symmetric. Therefore $\varepsilon_{N^{\prime}}=\varepsilon_{N^{\prime}}^{+}$. Now $\varepsilon_{N}^{+} \leqq \varepsilon_{N^{\prime}}^{+}$, and $\varepsilon_{N}^{+} \geqq \varepsilon_{N}=\varepsilon_{N^{\prime}}=\varepsilon_{N^{\prime}}^{+}$, so that $\varepsilon_{N}^{+}=\varepsilon_{N}$ for all $N>N^{\prime}$. Thus we conclude, as above, that for some constant $c$ : 
a) $\varepsilon_{N}^{+}=\varepsilon_{N-1}^{+}=\varepsilon_{N_{0}}^{+}$for $N \geqq N_{0}$,

b) $H_{N}(\omega)$ has no discrete spectrum in the symmetric subspace $\mathscr{D}^{+}$for $N \geqq N_{0}$,

c) $\varepsilon_{N}^{+}$is not an eigenvalue of $H_{N}(\omega)$ for $N \geqq c Z^{2}$, and

d) $N_{0} \leqq c Z^{2}$.

Theorem 1 implies that $\varepsilon_{N}=\varepsilon_{N_{0}}$ for all $N \geqq N_{0}$, so that $\varepsilon_{N}^{-} \geqq \varepsilon_{N} \geqq \varepsilon_{N_{0}}$. Thus there is a constant $A$ such that

$$
\varepsilon_{N}^{-} \geqq-A,
$$

i.e. the fermion ground state energy is bounded uniformly in $N$. This means that if Theorem 1 does not hold for fermions, then, at worst, $\varepsilon_{N-1}^{-}-\varepsilon_{N}^{-}$can be made arbitrarily small.

Our results extend to more general Hamiltonians than (1). In particular, Theorem 1 holds for molecules and for Hamiltonians in which potentials of the form $1 / r$ are replaced by $1 / r^{\gamma}$ where $0<\gamma<2$. For simplicity we give a detailed proof of Theorem $1 \mathrm{in} \mathrm{Sect.} \mathrm{II} \mathrm{only} \mathrm{for} \mathrm{the} \mathrm{Hamiltonian} \mathrm{given} \mathrm{by} \mathrm{(1).} \mathrm{Generalizations}$ are discussed in Sect. III.

We will use the following notation. Let $X=\left\{x=\left(x_{1} \ldots x_{N}\right)\right\}$ where $x_{j}=\left(\mathbf{r}_{j}, \alpha_{j}\right)$ denotes the space and (if necessary) spin coordinates, $\alpha_{j}$, of the $j^{\text {th }}$ particle. Integrals of the form $\int d x$ include both integration over $\mathbb{R}^{3 N}$ and summation over spin; and $\int d \hat{x}_{k}$ means integration over coordinates of all particles except the $k^{\text {th }}$. As usual $r_{k}=\left|\mathbf{r}_{k}\right|=\left|x_{k}\right|$ and $r_{j k}=\left|\mathbf{r}_{j}-\mathbf{r}_{k}\right|$. We now fix $p \geqq 2$ and define

and

$$
\begin{aligned}
|r|_{p} & =\left(\sum_{j=1}^{N} r_{j}^{p}\right)^{1 / p}, \\
\left|\hat{r}_{k}\right|_{p} & =\left(\sum_{j \neq k} r_{j}^{p}\right)^{1 / p}=\left(|r|_{p}^{p}-r_{k}^{p}\right)^{1 / p}, \\
\Omega_{k}(B) & =\left\{x:\left|\hat{r}_{k}\right|_{p}<B r_{k}\right\},
\end{aligned}
$$

$$
\Gamma_{k}(B)=\Omega_{k}(B)-\bar{\Omega}_{k}\left(\frac{B}{2}\right)=\left\{x: 1<r_{k} B\left|\hat{r}_{k}\right|_{p}^{-1}<2\right\} .
$$

In what follows, $p$ is fixed and the dependence of $\Omega_{k}(B)$ and $\Gamma_{k}(B)$ on $p$ is suppressed. The following properties of $\Omega_{k}(B)$ are extremely useful and easy to prove:

a) $x$ in $\Omega_{k}(B) \Rightarrow r_{j k}<(B+1) r_{k}$

b) $x$ in $\Omega_{k}(B) \Rightarrow r_{k}^{-1}<\left(B^{p}+1\right)^{1 / p}|r|_{p}^{-1}$

c) $x \notin \Omega_{k}(B) \Rightarrow\left|\hat{r}_{k}\right|_{p}^{-1} \leqq\left(B^{p}+1\right)^{1 / p}\left(B|r|_{p}\right)^{-1}$.

We can now sketch the main idea of our proof. In the cone $\Omega_{N}(B)$ one has by property (a) above

$$
-r_{N}^{-1}+\sum_{j=2}^{N} \omega r_{j N}^{-1} \geqq-r_{N}^{-1}+\omega(N-1)(B+1)^{-1} r_{N}^{-1}=\lambda r_{N}^{-1},
$$

where $\lambda=\omega(N-1)(B+1)^{-1}-1$ can be made positive by choosing $\omega$ or $N$ 
sufficiently large. It is then tempting to say

$$
\int_{\Omega_{N}(B)} \Psi H_{N} \Psi d x \geqq \int_{\Omega_{N}(B)} \Psi H_{N-1} \Psi d x \geqq \varepsilon_{N-1} \int_{\Omega_{N}(B)}|\Psi|^{2} d x .
$$

However, the second inequality holds only when $\Psi=0$ on $\partial \Omega_{N}(B)$ which is not true in general. Therefore, we will multiply $\Psi$ by a smoothing function $G$ so that $G \Psi=0$ on $X-\Omega_{N}(B)$. This will, of course, introduce an error in the kinetic energy, but we will show that this can be bounded outside the region $\left\{|x|:|r|_{p}<R\right\}$ for sufficiently large $R$. We will further show that for $B \sim N^{1 / p}$ the regions $\Omega_{k}(B)$ $(k=1 \ldots N)$ cover $X$. Thus, for sufficiently large $N$ and $R$, the question of whether or not $H_{N}$ has any discrete spectrum is determined entirely by $\int_{|r|_{p}<R} \Psi H_{N} \Psi d x$. We will show that $R \sim N^{3 / p}$ and that this is sufficient to show that $\int_{|r|_{p}<R} \Psi H_{N} \Psi d x>0$ for sufficiently large $N$.

The absence of discrete spectrum is therefore a consequence of the fact that the Coulomb repulsion $\sum_{j<k} \omega r_{j k}^{-1}$ dominates the attraction $-\sum_{j} r_{j}^{-1}$ when $N$ is large. Screening occurs when particles are far from the nucleus. The Coulomb repulsion limits the number of particles which can be within a ball of fixed radius. Therefore, when $N$ is large, the Coulomb repulsion forces particles away from the nucleus and into a region where screening gives an effective repulsive potential. This intuitive argument is independent of permutational symmetry and we expect our results to hold for both bosons and fermions. However we have only succeeded in proving that $\varepsilon_{N}^{-} \geqq \varepsilon_{N-1} \geqq-A$; we have not excluded the possibility that $\varepsilon_{N-1}^{-}>\varepsilon_{N}^{-} \geqq \varepsilon_{N-1}$. Further discussion of the fermion problem is given in Sect. III.

Results similar to Lemmas 2-6 have been obtained by Uchiyama [1] for $N=2$ and $\omega>1$, and by Zhislin [2] for $N-1>Z=1 / \omega$ using $p=2$. However, Zhislin's $R$ grows exponentially with $N$ and his proofs are considerably more complicated.

\section{Proof}

All of the smoothing functions we need will be defined in terms of a fixed function $g: \mathbb{R}^{+} \rightarrow \mathbb{R}$ such that

i) $g$ has a continuous second derivative,

ii) $g(t)=0$ if $t \leqq 1$,

iii) $g(t)=1$ if $t \geqq 2$,

iv) $0<g(t)<1$ if $1<t<2$, and

v) $M=\sup _{t}\left|g^{\prime}(t)\right|^{2}\left(1-g(t)^{2}\right)^{-1}<\infty$.

In order that $(\mathrm{v})$ be satisfied it is sufficient that $g \sim \exp \left(-(2-t)^{n}\right)$ near $t=2$.

We now give two Lemmas which will allow us to estimate the error in the kinetic energy which comes from our smoothing functions.

Lemma 2. Fix $B$ and $k$ and let $F$ be any positive function in $C^{2}(X)$ for which 
$\nabla_{j} F=0$ on $X-\Gamma_{k}$ for all $j=1 \ldots N$. Then

$$
\sum_{j=1}^{N} \int F^{2}\left|\nabla_{j} \Psi\right|^{2} d x=\sum_{j=1}^{N} \int\left|\nabla_{j}(F \Psi)\right|^{2} d x+\sum_{j=1}^{N} \int|\Psi|^{2} F \Delta_{j} F d x
$$

Proof. Let $\tilde{\nabla}_{k}=\underset{j \neq k}{\oplus} \nabla_{j}$. Then

$$
\begin{aligned}
\sum_{j \neq k} \int\left|\nabla_{j} F \Psi\right|^{2} d x= & \int|\Psi|^{2}\left|\tilde{\nabla}_{k} F\right|^{2} d x+\int F^{2}\left|\tilde{\nabla}_{k} \Psi\right|^{2} d x+2 \int F \tilde{\nabla}_{k} F \\
& \cdot\left(|\Psi| \tilde{\nabla}_{k}|\Psi|\right) d x .
\end{aligned}
$$

By Green's formula the last term on the right in (7) can be written as:

$$
\begin{gathered}
\frac{1}{2} \int d x \int_{r_{k} B / 2<\left|\hat{r}_{k}\right|_{p}<r_{k} B}\left(\tilde{\nabla} F^{2}\right) \cdot\left(\tilde{\nabla}|\Psi|^{2}\right) d \hat{x}_{k}=-\frac{1}{2} \int_{\Gamma_{k}(B)}|\Psi|^{2} \tilde{\Delta}_{k} F^{2} d x \\
=-\frac{1}{2} \int|\Psi|^{2} \tilde{\Delta}_{k} F^{2} d x=-\int|\Psi|^{2}\left|\tilde{\nabla}_{k} F\right|^{2} d x-\int|\Psi|^{2} F \tilde{\Delta}_{k} F .
\end{gathered}
$$

Substituting this in (7) gives

$$
\int\left|\tilde{\nabla}_{k}(F \Psi)\right|^{2} d x=\int F^{2}\left|\tilde{\nabla}_{k} \Psi\right|^{2} d x-\int|\Psi|^{2} F \tilde{\Delta}_{k} F d x .
$$

Similarly, one can show

$$
\int\left|\nabla_{k}(F \Psi)\right|^{2} d x=\int F^{2}\left|\nabla_{k} \Psi\right|^{2} d x-\int|\Psi|^{2} F \Delta_{k} F d x
$$

Combining (8) and (9) gives (6).

We now introduce the smoothing functions $G_{k}\left(x_{1} \ldots x_{N}\right)=g\left(r_{k} B\left|\hat{r}_{k}\right|_{p}^{-1}\right)$, where $g$ is defined above.

Lemma 3. Fix $k$ and $B$. Then for all $\Psi$ in $\mathscr{D}\left(H_{N}\right)$

$$
\begin{aligned}
-\sum_{j=1}^{N} \int \Psi \Delta_{j} \Psi d x= & \sum_{j=1}^{N} \int\left|\nabla_{j}\left(G_{k} \Psi\right)\right|^{2} d x+\sum_{j=1}^{N} \int\left|\nabla_{j}\left(\sqrt{1-G_{k}^{2} \Psi}\right)\right|^{2} d x \\
& -\sum_{j=1}^{N} \int|\Psi|^{2}\left|\nabla_{j} G_{k}\right|^{2}\left(1-G_{k}^{2}\right)^{-1} d x .
\end{aligned}
$$

Proof. Both $G_{k}$ and $\sqrt{1-G_{k}^{2}}$ satisfy the hypotheses of Lemma 2. Therefore

$$
\begin{aligned}
\sum_{j=1}^{N}-\int \Psi \Delta_{j} \Psi d x= & \sum_{j=1}^{N} \int G_{k}^{2}\left|\nabla_{j} \Psi\right|^{2} d x+\sum_{j=1}^{N} \int\left(1-G_{k}^{2}\right)\left|\nabla_{j} \Psi\right|^{2} d x \\
= & \sum_{j=1}^{N} \int\left|\nabla_{j}\left(G_{k} \Psi\right)\right|^{2} d x+\sum_{j=1}^{N} \int\left|\nabla_{j}\left(\sqrt{1-G_{k}^{2}} \Psi\right)\right|^{2} d x \\
& +\sum_{j=1}^{N} \int|\Psi|^{2}\left(G_{k} \Delta_{j} G_{k}+\sqrt{1-G_{k}^{2}} \Delta_{j} \sqrt{1-G_{k}^{2}}\right) d x
\end{aligned}
$$

The theorem then follows from the fact that for all $j$

$$
G_{k} \Delta_{j} G_{k}+\sqrt{1-G_{k}^{2}} \Delta_{j} \sqrt{1-G_{k}^{2}}=-\left|\nabla_{j} G_{k}\right|^{2}\left(1-G_{k}^{2}\right)^{-1} .
$$

We are now ready to estimate $E_{N}(\Psi) \equiv \int \Psi H_{N} \Psi d x$. We will let 


$$
\begin{aligned}
H_{N-1}^{k} & =-\sum_{j \neq k} \Delta_{j}-\sum_{j \neq k} r_{j}^{-1}+\sum_{\substack{i<j \\
i, j \neq k}} \omega r_{i j}^{-1} \\
& =H_{N}-\left(-\Delta_{k}-r_{k}^{-1}+\sum_{j \neq k} \omega r_{j k}^{-1}\right)
\end{aligned}
$$

and note that

$$
\int \Psi H_{N-1}^{k} \Psi d x \geqq \varepsilon_{N-1}\|\Psi\|^{2} \text { for all } \Psi \text { in } \mathscr{D}\left(H_{N}\right) .
$$

Lemma 4. Fix $p \geqq 2$ and $B \geqq 2$. Then for all $\Psi$ in $\mathscr{D}\left(H_{N}\right)$

$$
\begin{aligned}
& E_{N}(\Psi) \geqq \varepsilon_{N-1}\left\|G_{k} \Psi\right\|^{2}+E_{N}\left(\sqrt{1-G_{k}^{2}} \Psi\right)-2^{1+2 / p} M B^{2} \\
& \cdot \int|\Psi|^{2}|r|_{p}^{-2} d x+\lambda \int\left|G_{k} \Psi\right|^{2}|r|_{p}^{-1} d x,
\end{aligned}
$$

where $\lambda=\omega(N-1)(B+1)^{-1}-1$.

Proof. It follows from Lemma 3 that

$$
E_{N}(\Psi)=E_{N}\left(G_{k} \Psi\right)+E_{N}\left(\sqrt{1-G_{k}^{2}} \Psi\right)-\sum_{j=1}^{N} \int|\Psi|^{2}\left|\nabla_{j} G_{k}\right|^{2}\left(1-G_{k}^{2}\right)^{-1} d x .
$$

Now, using (12), one sees easily that

$$
E_{N}\left(G_{k} \Psi\right) \geqq \int\left(G_{k} \Psi\right) H_{N-1}^{k}\left(G_{k} \Psi\right) d x+\int\left|G_{k}\right|^{2}|\Psi|^{2}\left(-r_{k}^{-1}+\sum_{j \neq k} \omega r_{j k}^{-1}\right) d x
$$

Since $G_{k} \Psi=0$ for $x$ in $X-\Omega_{k}(B)$, the last term on the right in (17) becomes

$$
\int_{\Omega_{k}(B)}\left|G_{k}\right|^{2}|\Psi|^{2}\left(-r_{k}^{-1}+\sum_{j \neq k} \omega r_{j k}^{-1}\right) d x>\int \lambda\left|G_{k} \Psi\right|^{2} r_{k}^{-1} d x \geqq \lambda \int\left|G_{k} \Psi\right|^{2}|r|_{p}^{-1} d x,
$$

where we proceed first as in (5) and then use the fact that $r_{k}<|r|_{p}$. If we combine this with (13) applied to $G_{k} \Psi,(17)$ becomes

$$
E_{N}\left(G_{k} \Psi\right) \geqq \varepsilon_{N-1}\left\|G_{k} \Psi\right\|^{2}+\lambda \int\left|G_{k} \Psi\right|^{2}|r|_{p}^{-1} d x .
$$

We now need to estimate $\sum_{j=1}^{N} \int|\Psi|^{2}\left|\nabla_{j} G_{k}\right|^{2}\left(1-G_{k}^{2}\right)^{-1} d x$. We first note that $\nabla_{j} G_{k} \neq 0 \Rightarrow 1<r_{k} B\left|\hat{r}_{k}\right|_{p}^{-1}<2$. Then for $j \neq k$

$$
\begin{aligned}
\left|\nabla_{j} G_{k}\right|^{2}=\left|\frac{\partial}{\partial r_{j}} g\left(r_{k} B\left|\hat{r}_{k}\right|_{p}^{-1}\right)\right|^{2}= & \left|\hat{r}_{k}\right|_{p}^{-2}\left(r_{k} B\left|\hat{r}_{k}\right|_{p}^{-1}\right)^{2}\left(r_{j}\left|\hat{r}_{k}\right|_{p}^{-1}\right)^{2 p-2} \\
& \cdot\left|g^{\prime}\left(r_{k} B\left|\hat{r}_{k}\right|_{p}^{-1}\right)\right|^{2}
\end{aligned}
$$

so that for all $p \geqq 2$

$$
\sum_{j \neq k}\left|\nabla_{j} G_{k}\right|^{2}\left(1-G_{k}^{2}\right)^{-1} \leqq 4 M\left|\hat{r}_{k}\right|_{p}^{-2}\left|\hat{r}_{k}\right|_{p}^{-p} \sum_{j \neq k} r_{j}^{p}\left(r_{j} /\left|\hat{r}_{k}\right|_{p}\right)^{p-2} \leqq 4 M\left|\hat{r}_{k}\right|_{p}^{-2} .
$$

Similarly,

$$
\left|\nabla_{k} G_{k}\right|^{2}\left(1-G_{k}^{2}\right)^{-1} \leqq M B^{2}\left|\hat{r}_{k}\right|_{p}^{-2} .
$$

Combining these estimates and applying property (c) of $\Omega_{k}(B / 2)$, one finds that for all $B \geqq 2$ 


$$
\begin{aligned}
\sum_{j=1}^{N}\left|\nabla_{j} G_{k}\right|^{2}\left(1-G_{k}^{2}\right)^{-1} \leqq M\left(B^{2}+4\right)\left|\hat{r}_{k}\right|_{p}^{-2} & \leqq M\left(1+4 B^{-2}\right)\left(B^{p}+2^{p}\right)^{2 / p}|r|_{p}^{-2} \\
& \leqq 2^{1+2 / p} M B^{2}|r|_{p}^{-2}
\end{aligned}
$$

Substituting (18) and (19) in (16) gives (14).

We will prove our main theorem by repeated application of Lemma 4. To sketch the idea we consider $N=3$. Applying Lemma 4 twice one gets

$$
\begin{aligned}
E_{3}(\Psi) \geqq & \varepsilon_{2}\left(\left\|G_{1} \Psi\right\|^{2}+\left\|G_{2} \sqrt{1-G_{1}^{2}} \Psi\right\|^{2}\right)+E_{3}\left(\sqrt{1-G_{1}^{2}} \sqrt{1-G_{2}^{2}} \Psi\right) \\
& -8 M B^{2} \int|\Psi|^{2}|r|_{p}^{-2} d x+\lambda \int\left[\left|G_{1} \Psi\right|^{2}+G_{2}\left(1-G_{1}^{2}\right)|\Psi|^{2}\right]|r|_{p}^{-1} d x
\end{aligned}
$$

As we will see below, for suitable choices of $B$,

$$
\begin{aligned}
E_{3}\left(\sqrt{1-G_{1}^{2}} \sqrt{1-G_{2}^{2}} \Psi\right) \geqq & \varepsilon_{2}\left\|\sqrt{1-G_{1}^{2}} \sqrt{1-G_{2}^{2}} \Psi\right\|^{2} \\
& +\lambda \int\left(1-G_{1}^{2}\right)\left(1-G_{2}^{2}\right)|\Psi|^{2}|r|_{p}^{-1} d x,
\end{aligned}
$$

so that

$$
E_{3}(\Psi) \geqq \varepsilon_{2}\|\Psi\|^{2}+\int|\Psi|^{2}\left(\lambda|r|_{p}-8 M B^{2}\right)|r|_{p}^{-2} d x .
$$

We need to generalize this to arbitrary $N$ and to find conditions under which the last term can be made positive. Since $\lambda|r|_{p}-8 M B^{2}>0$ only for large $|r|_{p}$ we will also need to smooth around $\left\{x:|r|_{p} \leqq R\right\}$ for a suitable choice of $R$.

Lemma 5. Fix $p \geqq 2$ and let $B=2^{1+1 / p}(N-1)^{1 / p}$. Then for all $\Psi$ in $\mathscr{D}$,

$$
E_{N}(\Psi) \geqq \varepsilon_{N-1}\|\Psi\|^{2}+\int|\Psi|^{2}\left(\lambda|r|_{p}-2^{1+2 / p} M(N-1) B^{2}\right)|r|_{p}^{-2} d x
$$

where $\lambda=\omega(N-1)(B+1)^{-1}-1$.

Proof. Let $\Psi_{1}=\Psi, \Psi_{k+1}=\sqrt{1-G_{k}^{2}} \Psi_{k}(k=1 \ldots N-1)$. Then by repeated application of Lemma 4

$$
\begin{aligned}
E_{N}(\Psi) \geqq & \varepsilon_{N-1} \sum_{k=1}^{N}\left\|G_{k} \Psi_{k}\right\|^{2}+E_{N}\left(\Psi_{N}\right)-2^{1+2 / p} M(N-1) B^{2} \int|\Psi|^{2}|r|_{p}^{-2} d x \\
& +\lambda \sum_{k=1}^{N-1} \int\left|G_{k} \Psi_{k}\right|^{2}|r|_{p}^{-1} d x
\end{aligned}
$$

Now $\Psi_{k+1} \neq 0 \Rightarrow G_{k} \neq 1 \Rightarrow r_{k} B\left|\hat{r}_{k}\right|_{p}^{-1}<2$, and $\Psi_{N} \neq 0 \Rightarrow \Psi_{k} \neq 0$ for all $k \leqq N$. Thus, whenever $\Psi_{N} \neq 0$ we have

$$
r_{k}^{p} B^{p} \leqq 2^{p} \sum_{j \neq k} r_{j}^{p} \quad(k=1 \ldots N-1) .
$$

Adding these $N-1$ inequalities gives

$$
\left|\hat{r}_{N}\right|_{p}^{p}(B / 2)^{p} \leqq(N-2)\left|\hat{r}_{N}\right|_{p}^{p}+(N-1) r_{N}^{p},
$$

so that

$$
r_{N}^{p} \geqq\left[(B / 2)^{p}-(N-2)\right](N-1)^{-1}\left|\hat{r}_{N}\right|_{p}^{p}
$$

Now choose $B=2(2(N-1))^{1 / p}$. Then $N \geqq 2$ implies $B>2$ so that

$$
r_{N} \geqq\left(N(N-1)^{-1}\right)^{1 / p}\left|\hat{r}_{N}\right|_{p}>\left|\hat{r}_{N}\right|_{p}>B^{-1}\left|\hat{r}_{N}\right|_{p} .
$$


Thus $\left(-r_{N}^{-1}+\sum_{j=1}^{N-1} \omega r_{j N}^{-1}\right)>\lambda r_{N}^{-1}$ whenever $\Psi_{N} \neq 0$. Then, using (12) and (13) as in the proof of Lemma 4, one finds

$$
E_{N}\left(\Psi_{N}\right) \geqq \varepsilon_{N-1}\left\|\Psi_{N}\right\|^{2}+\lambda \int\left|\Psi_{N}\right|^{2}|r|_{p}^{-1} d x .
$$

Substituting (24) in (22) and using

$$
\sum_{j=1}^{N-1}\left|G_{k} \Psi_{k}\right|^{2}+\left|\Psi_{N}\right|^{2}=|\Psi|^{2}
$$

one finds

$$
E_{N}(\Psi) \geqq \varepsilon_{N-1}\|\Psi\|^{2}+\lambda \int|\Psi|^{2}|r|_{p}^{-1} d x-2^{1+2 / p} M(N-1) B^{2} \int|\Psi|^{2}|r|_{p}^{-2} d x .
$$

Remark. Let $R_{1}=2^{1+2 / p} M(N-1) B^{2} \lambda^{-1}$. If $\Psi=0$ whenever $|r|_{p}<R_{1}$, then Lemma 5 implies that $E_{N}(\Psi) \geqq \varepsilon_{N-1}\|\Psi\|^{2}$. Since $B<B+1 \leqq 3 B / 2$

$$
\omega 2^{-1-1 / p}(N-1)^{1-1 / p}>\lambda \geqq \omega 3^{-1} 2^{-1 / p}(N-1)^{1-1 / p}-1,
$$

so that when $N$ is large $\lambda>(\omega / 5)(N-1)^{1-1 / p}$ and $R_{1}<40 \cdot 2^{4 / p} M(N-1)^{3 / p} \omega^{-1}$.

Lemma 6. For every fixed $\omega$ there are constants $N_{\omega}$ and $c_{\omega}$ such that for every $N \geqq N_{\omega}$ and for every $\Psi$ in $\mathscr{D}\left(H_{N}\right)$ there is a $\Psi_{0}$ in $\mathscr{D}\left(H_{N}\right)$ such that

a) $\Psi_{0}=0$ whenever $r \geqq 2 R$ where $(2 / 5) c_{\omega}(N-1)^{3 / p}<R \leqq c_{\omega}(N-1)^{3 / p}$, and

b) $E_{N}(\Psi) \geqq \varepsilon_{N-1}\left(\|\Psi\|^{2}-\left\|\Psi_{0}\right\|^{2}\right)+\int \Psi_{0} H_{N}(\omega / 2) \Psi_{0} d x$.

Proof. Let $G_{0}(x)=g\left(|r|_{p} R^{-1}\right)$ and $\tilde{\nabla}=\bigoplus_{j=1}^{N} \nabla_{j}$. Then proceeding as in the proofs of Lemmas 2 and 3 one can show that

$$
\int G_{0}^{2}|\tilde{\nabla} \Psi|^{2} d x=\int\left|\tilde{\nabla} G_{0} \Psi\right|^{2} d x+\int|\Psi|^{2} G_{0} \tilde{\Delta} G_{0} d x
$$

and similarly for $\sqrt{1-G_{0}^{2}}$. Thus

$$
\begin{aligned}
-\sum_{j=1}^{N} \int \Psi \Delta_{j} \Psi d x= & \sum_{j=1}^{N} \int\left|\nabla_{j}\left(G_{0} \Psi\right)\right|^{2} d x+\sum_{j=1}^{N} \int\left|\nabla_{j} \sqrt{1-G_{0}^{2}} \Psi\right|^{2} d x \\
& -\sum_{j=1}^{N} \int_{R<|r|_{p}<2 R}\left|\nabla_{j} G_{0}\right|^{2}\left(1-G^{2}\right)^{-1}|\Psi|^{2} d x .
\end{aligned}
$$

Since $\left|\nabla_{j} G_{0}\right|=R^{-1} r_{j}^{p-1}|r|_{p}^{-(p-1)}\left|g^{\prime}\left(|r|_{p} R^{-1}\right)\right|$,

$$
\sum_{j=1}^{N}\left|\nabla_{j} G_{0}\right|^{2}\left(1-G^{2}\right)^{-1} \leqq R^{-2} M|r|_{p}^{-p} \sum_{j=1}^{N} r_{j}^{p}=R^{-2} M
$$

Let $\Psi_{0}=\sqrt{1-G_{0}^{2}} \Psi$ and $\Psi_{1}=G_{0} \Psi$. Then $\Psi_{0} \neq 0 \Rightarrow|r|_{p}<2 R$ and $\Psi_{1} \neq 0 \Rightarrow$ $|r|_{p}>R$. Using (27) and then applying Lemma 5 to $\Psi_{1}$ one finds

$$
\begin{aligned}
E_{N}(\Psi) \geqq & E_{N}\left(\Psi_{1}\right)+E_{N}\left(\Psi_{0}\right)-M R^{-2} \int_{R<|r|_{p}<2 R}|\Psi|^{2} d x \\
\geqq & \varepsilon_{N-1}\left\|\Psi_{1}\right\|^{2}+\lambda \int\left|\Psi_{1}\right|^{2}|r|_{p}^{-1} d x-2^{1+2 / p} M(N-1) B^{2} \int\left|\Psi_{1}\right|^{2}|r|_{p}^{-2} d x \\
& +\int \Psi_{0} H_{N}(\omega) \Psi_{0} d x-M R^{-2} \int_{R<|r|_{p}<2 R}|\Psi|^{2} d x
\end{aligned}
$$




$$
\begin{aligned}
\geqq & \varepsilon_{N-1}\left\|\Psi_{1}\right\|^{2}+\int\left|\Psi_{1}\right|^{2}|r|_{p}^{-2}\left[|r|_{p} \lambda / 2-2^{1+2 / p} M(N-1) B^{2}\right] d x \\
& +\int \Psi_{0} H_{N}(\omega / 2) \Psi_{0} d x \\
& +\int_{R<|r|_{p}<2 R}\left[(\lambda / 2)|r|_{p}^{-1}\left|\Psi_{1}\right|^{2}+\frac{1}{2} \sum_{i<k} \omega r_{j k}^{-1}\left|\Psi_{0}\right|^{2}-M R^{-2}|\Psi|^{2}\right] d x .
\end{aligned}
$$

Choose

$$
R=2^{2+2 / p} M(N-1) B^{2} \lambda^{-1} \text { with } B=2^{1+1 / p}(N-1)^{1 / p} .
$$

Since $\Psi_{1}=0$ when $|r|_{p}<R$, (29) implies

$$
\int\left|\Psi_{1}\right|^{2}|r|_{p}^{-2}\left(|r|_{p} \lambda / 2-2^{1+2 / p} M(N-1) B^{2}\right) d x>0 .
$$

Now use (25) to choose $N_{\omega}$ so that $N \geqq N_{\omega}$ implies

$$
\lambda>\left(\frac{\omega}{5}\right)(N-1)^{1-1 / p}>0 .
$$

Then $\omega N(N-1) / 4>\lambda$ for all $N \geqq N_{\omega}$, so that the last term on the right in (28) is bounded below by

$$
\begin{aligned}
& \int_{R<|r|_{p}<2 R}\left[(\lambda / 2)(2 R)^{-1}\left|\Psi_{1}\right|^{2}+4^{-1} \omega N(N-1)(4 R)^{-1}\left|\Psi_{0}\right|^{2}-M R^{-2}|\Psi|^{2}\right] d x \\
& \geqq \int_{R<|r|_{p}<2 R}\left[\lambda(4 R)^{-1}\left(\left|\Psi_{1}\right|^{2}+\left|\Psi_{0}\right|^{2}\right)-M R^{-2}|\Psi|^{2}\right] d x \\
& =\int_{R<|r|_{p}<2 R}|\Psi|^{2} R^{-1}\left(\lambda / 4-M R^{-1}\right) d x \\
& =\int_{R<|r|_{p}<2 R}|\Psi|^{2} \lambda 4^{-1} R^{-1}\left[1-\left(2^{2+4 / p}(N-1)^{1+2 / p}\right)^{-1}\right] d x>0 .
\end{aligned}
$$

Thus $N \geqq N_{\omega}$ implies

$$
E_{N}(\Psi) \geqq \varepsilon_{N-1}\left\|\Psi_{1}\right\|^{2}+\int \Psi_{0} H_{N}(\omega / 2) \Psi_{0} d x .
$$

Since $|\Psi|^{2}=\left|\Psi_{1}\right|^{2}+\left|\Psi_{0}\right|^{2}$, this gives (26). To complete the proof we note that (25), and (29) imply that for $N \geqq N_{\omega}$

$$
\omega^{-1} 2^{5+5 / p} M(N-1)^{3 / p}<R \leqq \omega^{-1} 2^{4+4 / p} \cdot 5(N-1)^{3 / p} .
$$

This gives (a) if $c_{\omega}=80 \cdot 2^{4 / p} M \omega^{-1}$.

Proof of Theorem 1. By Lemma 6, if suffices to show that

$$
\int_{|r|_{p}<2 R} \Psi_{0} H_{N}(\omega / 2) \Psi_{0} d x \geqq \varepsilon_{N-1}\left\|\Psi_{0}\right\|^{2}
$$

where $\quad H_{N}(\omega / 2)=H_{N}^{0}+\frac{1}{2} \sum_{j<k} \omega r_{j k}^{-1}, H_{N}^{0}=-\sum_{j=1}^{N}\left(\Delta_{j}+r_{j}^{-1}{ }^{-1}\right), \quad$ and $\quad R \quad$ is given by (29). It is well-known that $H_{0} \geqq-N / 4$ and, as before, $|r|_{p}<2 R \Rightarrow r_{j k}<4 R$. Therefore,

$$
\begin{aligned}
\int_{|\boldsymbol{r}|_{p}<2 R} \Psi_{0} H_{N}(\omega / 2) \Psi_{0} d x & \geqq\left[-\frac{N}{4}+\omega N(N-1)(16 R)^{-1}\right]\left\|\Psi_{0}\right\|^{2} \\
& \geqq\left(\frac{N}{16}\right)\left[-4+\omega c_{\omega}^{-1}(N-1)^{1-3 / p}\right]\left\|\Psi_{0}\right\|^{2},
\end{aligned}
$$


where the last inequality follows from (32) for $N \geqq N_{\omega}$. When $p>3$, (33) can be made arbitrarily large by choosing $N$ sufficiently large. Let $p=4$ and choose $N_{0} \geqq N_{\omega}$ so that $\left(N_{0} / 16\right)\left[\omega c_{\omega}^{-1}(N-1)^{1 / 4}-4\right]>\varepsilon_{N-1}$. Then by Lemma 6

$$
E_{N}(\Psi)>\varepsilon_{N-1}\left(\|\Psi\|^{2}-\left\|\Psi_{0}\right\|^{2}\right)+\varepsilon_{N-1}\|\Psi\|_{0}^{2}=\varepsilon_{N-1}\|\Psi\|^{2}
$$

\section{Remarks and Generalization}

\section{A. Dependence of $N_{0}$ on $Z$}

We consider the case $W=1$ so that $\omega=Z^{-1}$. By the remark following Lemma 5 , it follows that the $N_{\omega}$ of Lemma 6 grows no worse than $Z^{p / p-1}$. In fact (25) implies $\left(N_{\omega}-1\right) \leqq\left[15 /\left(5 \cdot 2^{-1 / p}-3\right)\right]^{p / p-1} Z^{p / p-1}$. Since $p$ can be chosen arbitrarily large, we find $N_{\omega}<8 Z+1$. Thus, we conclude that for large $Z$ the dependence of $N_{0}$ on $Z$ is determined by (33). Since $\varepsilon_{N-1}$ is not known exactly, we will find $N_{0}$ large enough to satisfy $-1+\omega\left(4 c_{\omega}\right)^{-1}(N-1)^{1-3 / p} \geqq 0$. Thus

$$
\left(N_{0}-1\right)^{(p-3) / p}=4 c_{\omega} \omega^{-1}=5 \cdot 2^{6} 2^{4 / p} M Z^{2} .
$$

when $p=4$, this gives $N_{0} \leqq 5^{4} 2^{28} M^{4} Z^{8}$. However, since $p$ can be made arbitrarily large, we obtain the better estimate

$$
N_{0}-1 \leqq 320 M Z^{2}
$$

\section{B. Molecules}

Our results remain true if we replace $-Z r_{k}^{-1}$ in $H_{N}(W, Z)$ by $-\sum_{\ell=1}^{L} Z_{\ell}\left|\mathbf{r}_{k}-\mathbf{R}_{\ell}\right|^{-1}$ where $\mathbf{R}_{\ell}$ are fixed. Choose $\tilde{R}$ so that $\left(B^{p}+1\right)^{-1 / p} \tilde{R} / 2=\max \left\{R_{1} \ldots R_{L}\right\}$. Then if $|r|_{p} \geqq \tilde{R}$ and $x$ in $\Omega_{k}(B), R_{\ell} \leqq\left(B^{p}+1\right)^{-1 / p}|r|_{p} / 2<r_{k} / 2$, and

$$
\begin{aligned}
-\sum_{\ell=1}^{L} Z_{\ell}\left|\mathbf{r}_{k}-\mathbf{R}_{\ell}\right|^{-1}+W \sum_{j \neq k} r_{j k}^{-1} & \geqq-2 \sum_{\ell=1}^{L} Z_{\ell} r_{k}^{-1}+W(N-1)(B+1)^{-1} r_{k}^{-1} \\
& =Z \lambda r_{k}^{-1},
\end{aligned}
$$

where $Z=2 \sum_{\ell=1}^{L} Z_{\ell}$ and $\lambda=W Z^{-1}(N-1)(B+1)^{-1}-1$ as before. Since $\tilde{R} \sim B \sim$ $(N-1)^{1 / p}$ and $R \sim B^{3} \sim(N-1)^{3 / p}$ in Lemma $6, R>\widetilde{R}$ for sufficiently large $N$. Let $\Psi_{1}$ be as in Lemma 6 . Then $\Psi_{1}=0$ for $r<\widetilde{R}<R$ and Lemmas 4 and 5 remain valid when applied to $\Psi_{1}$. Thus Lemma 6 holds with $\omega=W\left(2 \sum_{\ell=1} Z_{\ell}\right)^{-1}$ and a possibly larger $N_{\omega}$.

\section{Other Power Law Potentials}

Our methods can easily be generalized to show that the Hamiltonian

$$
H_{N}^{\gamma}(\omega)=-\sum_{j=1}^{N} \Delta_{j}-\sum_{j=1}^{N} r_{j}^{-\gamma}+\omega \sum_{j<k} r_{j k}^{\gamma}
$$

also satisfies Theorem 1 , when $0<\gamma<2$. 
Since $x$ in $\Omega_{k}(B)$ implies that $-r_{k}^{-\gamma}+\omega \sum_{j \neq k} r_{j k}^{-\gamma} \geqq \lambda_{\gamma} r_{k}^{-\gamma}$, where $\lambda_{\gamma}=\omega(N-1)$ $(B+1)^{-\gamma}-1$, Lemmas 4 and 5 remain valid if $\lambda \int\left|G_{k} \Psi\right|^{2}|r|_{p}^{-1} d x$ is replaced by $\left.\lambda_{\gamma} \int \mid G_{k} \Psi\right\}^{2}|r|_{p}^{-\gamma} d x$. Then in the proof of Lemma 6, (30) becomes $\int\left|\Psi_{1}\right|^{2}|r|_{p}^{-2}$ $\left(|r|_{p}^{2-\gamma} \lambda_{\gamma}-2^{2+2 / p} M(N-1) B^{2}\right) d x>0 \quad$ if $\quad R^{2-\gamma}=2^{2+2 / p} M(N-1) B^{2} \lambda_{\gamma}^{-1}=$ $c_{1}(N-1)^{(2+\gamma) / p}$ for some constant $c_{1}$. The last integral in (28) can again be made positive as in (31) since

$$
\left(\lambda_{\gamma} / 4\right) R^{-\gamma}-M R^{-2}=\left(\lambda_{\gamma} / 4\right) R^{-\gamma}\left[1-\left(2^{2+4 / p}(N-1)^{1+2 / p}\right)^{-1}\right]>0 .
$$

Thus Lemma 6 holds with $R \leqq c_{2}(N-1)^{1 / s}$ where $s=p(2-\gamma) /(2+\gamma)$ for some constant $c_{2}$. We complete the proof as before using

$$
\begin{aligned}
\int_{|r|_{p}<2 R} \Psi_{0} H_{N}(\omega / 2) \Psi_{0} d x & \geqq\left(-\frac{N}{4}+\omega N(N-1) 16^{-1} R^{-\gamma}\right)\left\|\Psi_{0}\right\|^{2} \\
& =\frac{N}{4}\left(-1+c_{3}(N-1)^{1-\gamma / s}\right)\left\|\Psi_{0}\right\|^{2},
\end{aligned}
$$

for some positive constant $c_{3}$. For each fixed $\gamma<2$, we can choose $p$ so that $s>\gamma$ and (35) can be made arbitrarily large.

\section{Increasing the Interaction}

If $N$ is fixed, $H_{N}(W, Z)$ has no bound states if the nuclear charge $Z$ is sufficiently small or the interaction parameter $W$ is sufficiently large. To be precise, for each fixed $N$ there is an $\omega_{0}$ such that $\omega=W Z^{-1}>\omega_{0}$ implies that $H_{N}(\omega)$ has no discrete spectrum.

When $N=2$ this result was first proven by Uchiyama [15]. Since the fermion ground state is always a singlet when $N=2, \varepsilon_{2}^{+}=\varepsilon_{2}^{-}=\varepsilon_{2}$ so that both bosons and fermions have the same critical $\omega_{0}$. Using a perturbation expansion in $\omega$, Stillinger [16] has estimated $\omega_{0} \simeq 1.0975$. Ruskai [17] has used Hill's techniques $[4,5]$ to obtain the less accurate, but rigorous bound $\omega_{0}<1.343$.

We now sketch the proof that $H_{N}(\omega)$ has no discrete spectrum for sufficiently large $\omega$. As before, the proof for bosons follows from the proof for particles without any permutational symmetry restrictions. Lemmas 2-5 do not depend $c n$ the value of $\omega$. Lemma 6 holds for arbitrary $N$ if $\omega_{1}$ is chosen so that $\lambda>(\omega / 5)(N-1)^{1-1 / p}$ whenever $\omega>\omega_{1}$ as in (25). To complete the proof, we write (33) as

$$
\int_{|r|_{p}<2 R} \Psi_{0} H_{N}(\omega / 2) \Psi_{0} d x \geqq(N / 4)\left[-1+\omega^{2} c_{N}\right]\left\|\Psi_{0}\right\|^{2}
$$

for some constant $c_{N}$. Since (36) can be made arbitrarily large by choosing $\omega$ sufficiently large, the argument following (33) can be used to show $E_{N}(\Psi) \geqq$ $\varepsilon_{N-1}\|\Psi\|^{2}$ for $\omega$ sufficiently large.

\section{E. Fermions}

We now indicate which portions of our proof in Sect. II remain valid for fermions, i.e. if we restrict $\Psi$ to $\mathscr{D}^{-}$and replace $\varepsilon_{N}$ by $\varepsilon_{N}^{-}$. Lemmas 2 and 3 obviously do 
not depend on permutational symmetry. Before discussing Lemmas 4-6, we note that $G_{k}$ is symmetric with respect to the interchange $i \leftrightarrow j$ provided $i \neq k$ and $j \neq k$, but not with respect to $i \leftrightarrow k$. Therefore $G_{k} \Psi$ is antisymmetric in the coordinates of the $N-1$ particles $(1, \ldots, k-1, k+1, \ldots, N)$, so that

$$
\int\left(G_{k} \Psi\right) H_{N-1}^{k} G_{k} \Psi d x \geqq \varepsilon_{N-1}^{-}\left\|G_{k} \Psi\right\|^{2} \text {. }
$$

This is sufficient to extend the proof of Lemma 4 to fermions.

The proof of Lemma 5 does not, unfortunately, hold for fermions. The problem is that $\Psi_{k}=\prod_{j=1}^{k-1} \sqrt{1-G_{j}^{2}} \Psi$ and $G_{k} \Psi_{k}$ will not be antisymmetric in general so that Lemma 4 cannot be applied to $\Psi_{k}$. The functions $G_{k} \Psi_{k}$ are antisymmetric within the clusters $(1, \ldots, k-1)$ and $(k+1, \ldots, N)$ but not with respect to interchanges between clusters. Therefore (13) becomes $\int G_{k} \Psi_{k} H_{N-1}^{k} G_{k} \Psi_{k} \geqq \varepsilon_{N-1}^{k}\left\|G_{k} \Psi_{k}\right\|^{2}$ where $\varepsilon_{N-1}^{k}$ is defined by taking the infimum in (2) over the subspace $\mathscr{D}^{k}\left(H_{N-1}\right)$ consisting of functions antisymmetric within the clusters $(1, \ldots, k-1)$ and $(k+1, \ldots, N)$. It would suffice to show $\varepsilon_{N-1}^{k} \geqq \varepsilon_{N-1}^{-}$, but we know of no reason to expect this to hold in general. We note, however, that $\mathscr{D}^{k}$ is an invariant subspace of $H_{N-1}$ containing $\mathscr{D}^{-}$. Choose $N^{\prime}$ so that $\varepsilon_{N^{\prime}}^{k}$ is an eigenvalue of $H_{N^{\prime}}$ and $\varepsilon_{N^{\prime}+1}^{k}=\varepsilon_{N^{\prime}}^{k}$. If $N^{\prime}>3$ and this ground state happens to be unique, it must be antisymmetric since there are no totally symmetric functions in $\mathscr{D}^{k}$. Then by the same argument used to show that $\varepsilon_{N}^{+}=\varepsilon_{N}$, we could conclude $\varepsilon_{N-1}^{-}=\varepsilon_{N-1}^{k}$. Unlike the symmetric case, however, we know of no physical or mathematical reason which would justify the assumption that $H_{N}$ has a unique ground state on $\mathscr{D}^{k}\left(H_{N}\right)$. Therefore, we cannot conclude that $\varepsilon_{N}^{k}=\varepsilon_{N}^{-}$, except by occasional accident.

Lemma 6 and the proof of Theorem 1 are invalid for fermions only because they depend on Lemma 5. In fact, our entire analysis extends to fermions except for the application of (13) to $G_{k} \Psi_{k}$. If a suitable extention of Lemma 5 could be found, Lemma 6 would also hold. The completion of Theorem 1 can even be modified, if necessary, to accommodate a different dependence of $R$ on $N$. Since $\varepsilon_{N}^{-}$is bounded uniformly in $N$, we can use (4) to replace (33) by

$$
\begin{aligned}
& \int \Psi_{0} H_{N}(\omega / 2) \Psi_{0} \geqq \int \Psi_{0} H_{N}(\omega / 4) \Psi_{0} d x+(\omega / 4) \sum_{j<k} \int_{|r|} \leqq 2 R \\
& \Psi_{0} r_{j k}^{-1} \Psi_{0} d x \\
& \geqq(-A+\omega N(N-1) /(32 R))\left\|\Psi_{0}\right\|^{2},
\end{aligned}
$$

which can be made arbitrarily large by making $N$ large, provided that $R$ grows more slowly than $N^{2}$. In the boson case (33) needed $R$ growing more slowly than $N$, which was satisfied by choosing $p>3$ since Lemma 5 implied $R \sim(N-1)^{3 / p}$. Because the boson result implies uniform boundedness of $\varepsilon_{N}^{-}$, (37) can tolerate $R \sim N^{t}$ for some $t<2$. Thus a weaker version of Lemma 5 would suffice in the fermion case.

If the fermion proof could be completed with $R \sim N^{k / p}$ for some fixed $k$ and arbitrarily large $p$, then one could similarly improve the bound on $N_{0}$. We first use the well-known fact that the eigenfunctions of $H$ are Slater determinants of the hydrogenic eigenfunctions, $\phi_{n}^{k}\left(k=1, \ldots, q n^{2}\right)$, of $-\Delta+r^{-1}$ which have eigenvalues $\lambda_{n}^{k}=-1 / 4 n^{2}\left(k=1, \ldots, q n^{2}\right)$ where $q$ is the number of spin states. Then there 
is an $n_{0}$ such that

$$
N \geqq \sum_{n=1}^{n_{0}-1} q n^{2} \geqq c_{1} n_{0}^{3}
$$

and the ground state energy of $H^{0}$ is $\geqq-\sum_{n=1}^{n_{0}} q n^{2} / 4 n^{2} \geqq-\left(q c_{1}^{1 / 3} / 4\right) N^{1 / 3}$. Then (33) could be replaced by

$$
\int_{|r|_{p}<2 R} \Psi_{0} H_{N}(\omega / 2) \Psi_{0} d x \geqq\left[-\alpha N^{1 / 3}+\beta Z^{-2} N(N-1)^{1-k / p}\right]\left\|\Psi_{0}\right\|^{2}
$$

for some constants $\alpha$ and $\beta$. Then we could conclude, as in Sect. A above, that $N_{0} \leqq B Z^{6 / 5}$ for some constant $B$.

Acknowledgements. It is a pleasure to thank Professors R. Benguria, T. Hoffmann-Ostenhof, and J. K. Percus for their encouragement and for stimulating discussions. This problem was suggested independently by Professors E. Lieb and L. Spruch. The final version of this paper was written while I was guest professor at the University of Vienna. I am especially grateful to Professors K. Case and W. Thirring for the hospitality extended to me by the Rockefeller University and by the University of Vienna, respectively.

\section{References}

1. Uchiyama, J.: Publ. Res. Inst. Math. Sci. Kyoto A5, 51-63 (1969)

2. Zhislin, G. M.: Teor. Mat. Fiz. 7, 332-341 (1971); (Theor. Math. Phys. 7, 571-578 (1971))

3. Yafaev, D. R.: Funkt. Anal. i Prilozeh. 6, 103-104 (1972) (Funt. Anal. Appl. 6, 349-350 (1972))

4. Hill, R. N.: Phys. Rev. Lett. 38, 643-646 (1977); J. Math. Phys. 18, 2316-2330 (1977)

5. Hill, R. N.: In: Mathematical Problems in Theoretical Physics, pp. 52-56. Berlin, Heidelberg, New York: Springer 1980.

6. Grosse, H., Pittner, L.: preprint

7. Hill, R. N.: private communication. See also [5]

8. Grosse, H.: J. Phys. A10, 711-716 (1977)

9. Yafaev, D.: Theor. Math. Phys. (USSR) 27, 328-330 (1977)

10. Klaus, M., Simon, B.: Commun. Math. Phys. 78, 153-168 (1980)

11. Hunziker, W.: Helv. Phys. Acta 39, 451-462 (1966)

12. van Winter, C.: Mat.-Fys. Skr. Danske Vid. Selsk. 1 (8), 1-60 (1964)

13. Zhislin, G.: Tr. Mosk. Mat. Obs. 9, 81-128 (1960)

14. Reed, M., Simon, B.: Methods of modern mathematical physics IV. Analysis of operators, New York: Academic Press 1978

15. Uchiyama, J.: Publ. Res. Inst. Math. Sci. Kyoto A6, 189-192 (1970)

16. Stillinger, F. H.: J. Chem. Phys. 45, 3623-3631 (1966)

17. Ruskai, M. B.: (unpublished)

18. Vugal'ter, S. A., Zhislin, G. M.: Teor. Mat. Fiz. 32, 70-87 (1977), (Theor. Math. Phys. 32, 602-614 (1977))

Communicated by E. Lieb

Received February 13, 1981 
